\title{
Extended Cellular Automata Based Model for Simulating Multi-Scale Urban Growth Using GIS
}

\author{
Yongjiu Feng Xiaohua Tong Miaolong Liu \\ Department of Surveying and Geo-informatics, Tongji University, Shanghai 200092, P. R. China
}

\begin{abstract}
Cellular Automata (CA) has attracted growing attention in urban simulation because their capability in spatial modelling is not fully developed in GIS. A "bottom-up" model is proposed for simulating complex urban system based on the integration of extended cellular automata (ECA) and geographic information system (GIS). The entity framework of the model is established for multi-scale land use changes and urban growth. The model has been successfully applied to the simulation of three urban areas at different scales: Pudong New District, Lujiazui Finance \& Trade Zone and Sanlin Town in Shanghai, China.
\end{abstract}

Keywords: Extended cellular automata, Geographical information system, Urban growth, Multi-scale dynamic simulation

\section{Introduction}

A CA model is a dynamic model with local interactions to reflect evolution of the system, where space and time are considered as discrete units and space is often represented as a regular lattice of two dimensions [1]. Because of the strong ability to represent non-linear, spatial and stochastic processes of CA models [2], it does not take long for geographers to apply CA to simulate land use change, urban development and other changes of geographical phenomena.

The application of CA in geographic phenomena can be traced back to 1960s when Chapin and Weiss from North Carolina State University, USA have used this discrete dynamic model in land use changes studies [3]. After several decades, the application of CA in geography has attained remarkable progresses. Among the preeminent researchers, Helen Couclelis is the greatest one who built the fundamental framework of CA application on geography [4]-[6], and explored the integration of Geographical Information System (GIS) and CA [7]. In 1990s, Roger White and Guy Eengelen simulated land use changes using $\mathrm{CA}$, such as urban growth of Cincinnati city of USA and the impact of global climate changes on land cover[8]-[9].

Studies of CA application on geography also attained some progresses at home. Cao Zhongchu et al. explored the CA model and its application on geography and traffic [10]-[11], and Fulong Wu integrated CA with Multi-criteria Evaluation model (MEC) in a unified user interface using AML and C language in ArcInfo GIS environment [12]. Li Xia et al. simulated and predicted the urban sustainable development using CA [13], and proposed a dynamic constrained CA model for simulating complex urban system [14] and an improved minimum-distance method for mining CA transition rules [15]. Zhou Chenghu et al. simulated and predicted the urban dynamic growth of Ann Arbor city of USA using GeoCA-Urban model [16]. Wang Chunfeng studied the simulation of Xi'an city of China using remote sensing and CA with the vector data of the city [17]. Liu Miaolong et al. put forward a prototype of urban simulation model based on the theories and methodologies of CA and Multi-Agent System [18].

Although studies of CA on spatial complex modeling obtained many exciting results at home, there are few works on the integration of CA and GIS\& remote sensing for multi-scale modeling and application. In this paper an improved evolution model for multi-scale urban land use changes and urban growth is proposed based on the integration of ECA model and GIS \& RS. As cases study, the urban dynamic growth of multi-scale areas are simulated and predicted in Pudong New District, Lujiazui Finance \& Trade Zone and Sanlin Town in Shanghai, China.

\section{Theories and methods}

\subsection{Cellular automata}

CA is a discrete model studied in computability theory, geography, mathematics, and theoretical biology. CA is a dynamic system defined on a cells space with finite discrete states which evolve according to a certain transition rules of neighboring states on 
discrete time scale. Also, CA is a model framework based on the modeling idea of "part-whole" and "bottom-up" processes.

A CA system usually consists of four elements: cells, states, neighborhoods and rules. The state of a cell changes according to transition rules defined in terms of neighborhood functions. Any n-dimension CA (n-CA) is a quaternion ( $\left.Z^{n}, S, N, f\right)$, where $Z^{n}$ is n-dimension Euclidean space; $S$ is a set with finite states: $S=\left\{S_{1}, S_{2}, \ldots, S_{i}, \ldots, S_{k}\right\}, S_{i}$ represents the state of ith CA; $N$ is neighborhood of center cell; $f$ is CA transition rules. Using set language, $\mathrm{CA}$ is given by:

$$
\mathrm{S}^{\mathrm{t}+1}=\mathrm{f}\left(\mathrm{S}^{\mathrm{t}}, \mathrm{N}\right)
$$

Here, $S$ is a finite set, representing states of cells; $f$ is partial transition rules; $N$ is neighborhood and $t$ is discrete modeling time. Formula (1) indicates that the state of a cell at time $t+1$ determined by the cell state, states of neighborhood cells and transition rules at time $t$.

However, there are many limitations on the application of CA (e.g. conflict of simplicity with facticity, and problems of transition rules). In order to resolve this problem, ECA (Extended cellular automata) was proposed [16]. The ECA model is put forward by extending original CA model (e.g. extending definition of neighborhood, states of cells and transition rules).

\subsection{Entity framework of urban simulation}

Traditional GIS describes spatial distribution of geography entity and phenomena well in a digital manner. However, the GIS techniques have limitations in modelling changes in the landscape over time, and lack the ability to characterize the temporal information and space-time relation. One of the solutions to these defects is the integration of GIS and ECA [16]. Based on microcosmic dynamic simulation theory, CA (a kind of microcosmic discrete simulation methods) is introduced into GIS environment, establishing a model incorporated space and time for dynamic process simulation and analysis. This model makes up ECA's inability on space and ignoring of inseparability rules of time and space in spatialtemporal process simulation in traditional geography.

Extending the original CA model at cell states, cell space, neighbor definition, evolution rules and discrete time, an entity framework of land use changes and urban growth simulation is proposed based on geographical information system (see Fig.1).

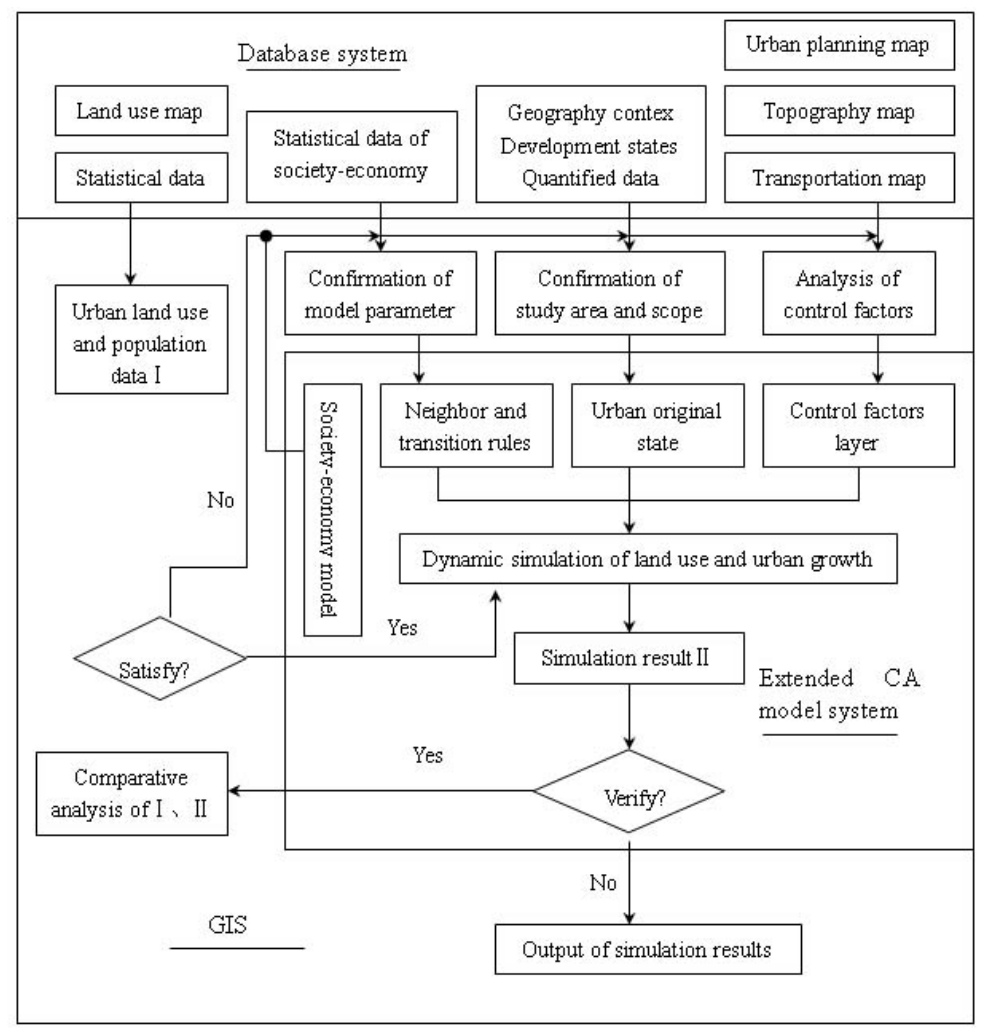

Fig.1: Entity framework of land use changes and urban growth simulation based on integrated GIS and ECA. 
In this model framework four parts are included: database system (DBS), geographical information system (GIS), society-economy model (SEM) and extended cellular automata (ECA) where ECA holds core situation used for modeling land use changes and urban growth dynamic changes. In this model SEM couples with CA and the model parameters are adjusted according to the development of society and economy. GIS, at the accessorial condition, can analyze and process all kinds of spatial and statistical data, and can simulate the urban dynamic growth process and demonstrate land use dynamic changes in the GIS environment. Analysis of the differences of land use of different period enables the study of laws of land use changes and urban growth. Through the visual and mapping function tools, the simulation results and graphs can be outputted.

\section{Implementation and discussion}

\subsection{Backgrounds of study areas}

In this paper three areas at different scales are studied: Pudong New District, Lujiazui Finance \& Trade Zone, and Sanlin Town. Lujiazui and Sanlin are two different areas of Pudong New District, Shanghai, China.

Pudong New District, located in east Shanghai, with total area $533.45 \mathrm{~km}^{2}$ and 21 Streets (Towns), has Yangtze River shoreline length 46.43km and Huangpu River shoreline length $43.5 \mathrm{~km}$. At the important position of "reform and opening" policies of China, Pudong city experienced a drastic urban expansion and growth. With the rapid growth of population, industry and infrastructure, this developing trend will continue for several decades.

Lujiazui Finance \& Trade Zone including 3 Streets, located in central Pudong New District, is the only national development area named as "Finance \& Trade” in China. Being one of the important parts of Shanghai CBD, the land use of the area has changed dramatically since 1990 .

Sanlin Town is located in the southwest of Pudong New District with area $31.28 \mathrm{~km}^{2}$. Much of the land cover in this region is agriculture land which will suffer the experience of change to urban land use in the future.

\subsection{Data and model parameters}

In this GIS-based ECA model, spatial and statistical data are vital for urban growth simulation which used for determining the model parameters of the seeds points, adjusting the model parameters and controlling the factors layer. At the same time, society-economy statistical data, geography context and development status data, and quantification data are needed for the simulation and predication. In the studies of the three regions at different scales, multi-source data are collected (e.g. land use data in past years, the latest transportation and traffic data, permanent population data and the number of employees).

Because of data formats differences of different data sources, GIS is used to unify the data format and the time and space scope of the data. In the model, only three types of land use are included which are housing land, commerce land and industry land.

The integration of GIS and ECA model is demonstrated by: (1) using GIS to found the background database needed by ECA, for example, using the ArcView GIS software to capture the data of land use map and transportation map of the study area; (2) Using ArcView GIS to unify the space of model; (3) Using GIS to establish dynamic analysis modeling system for urban growth; and (4) Using ECA to extend the spatial dynamic analysis functions. Embedding GIS functions in ECA model, the integration of GIS and ECA model is achieved in practice.

In the integration (GIS and ECA) model, seeds points are the original state of urban development. Therefore, the distribution of growth points should reflect the growth factually. According to the development of Pudong, Lujiazui and Sanlin, the model parameters of the three regions are setup respectively.

\subsection{Results of simulating and predicting}

With the year 2000 as original time of the model, urban growth are simulated and predicted in Pudong at model time $\mathrm{t}=10,20,30$ and 50(the year 2010, 2020, 2030 and 2050 respectively) and in Lujiazui and Sanlin at model time $\mathrm{t}=3,10,20$ and 50 (the year 2003, 2010, 2020 and 2050 respectively) (see Fig.2, where $a$ is Pudong, $b$ is Lujiazui and $c$ is Sanlin. In the legend $\mathrm{H}$ is housing land, $\mathrm{C}$ is commerce land, I is industry land, V is vacant land, SV is street vertex, and $\mathrm{O}$ is others land).

Fig.2 shows clearly the primary characteristics and spatial differences among the three regions at different scales. 


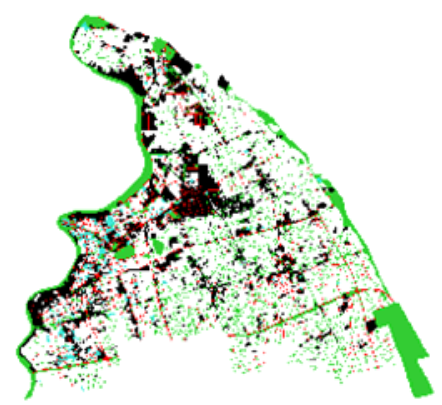

a1) 2000, Pudong Model Time: $\mathrm{t}=0$

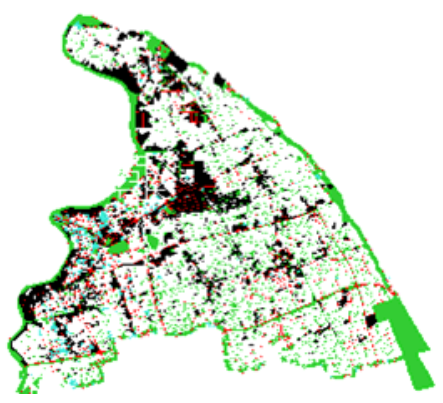

a2) 2010, Pudong Model Time: $\mathrm{t}=10$

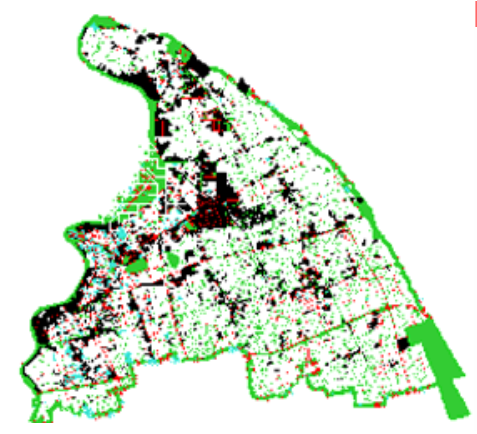

a3) 2020, Pudong Model Time: $\mathrm{t}=20$

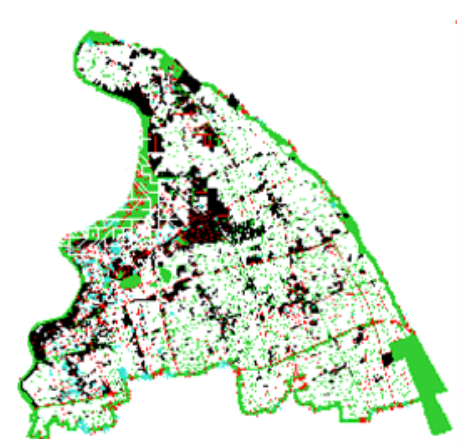

a4) 2030, Pudong

Model Time: $\mathrm{t}=30$

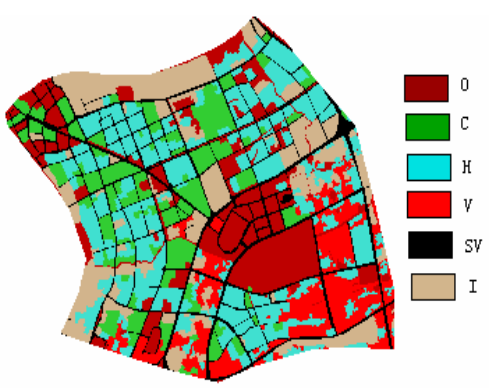

b1) 2000, Lujiazui Model Time: $\mathrm{t}=0$

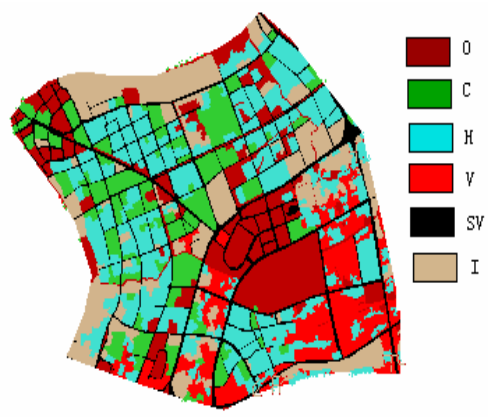

b2) 2003, Lujiazui Model Time: $\mathrm{t}=3$

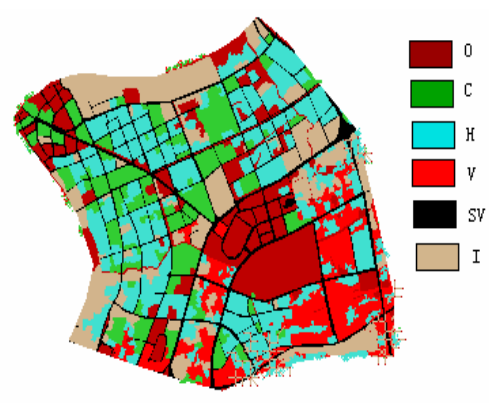

b3) 2010, Lujiazui Model Time: $\mathrm{t}=10$

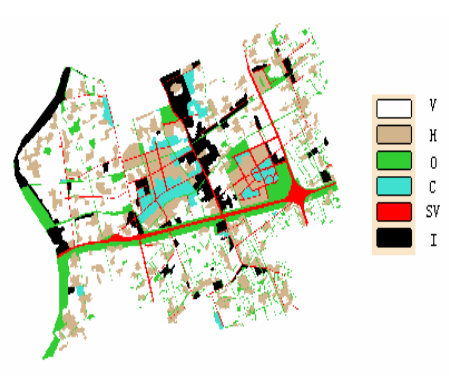

c1) 2000, Sanlin Model Time: $\mathrm{t}=0$

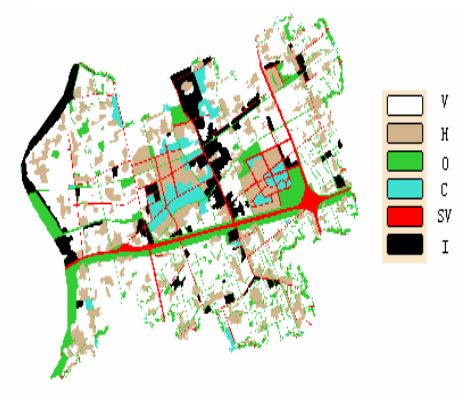

c3) 2003, Sanlin

Model Time: $\mathrm{t}=3$

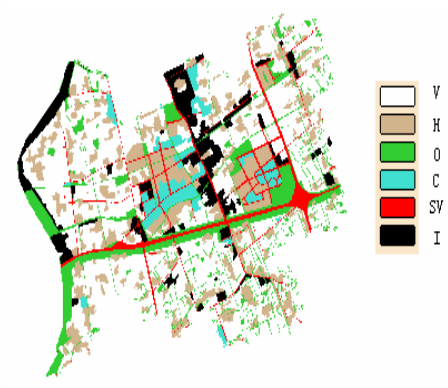

c3) 2010, Sanlin Model Time: $\mathrm{t}=10$

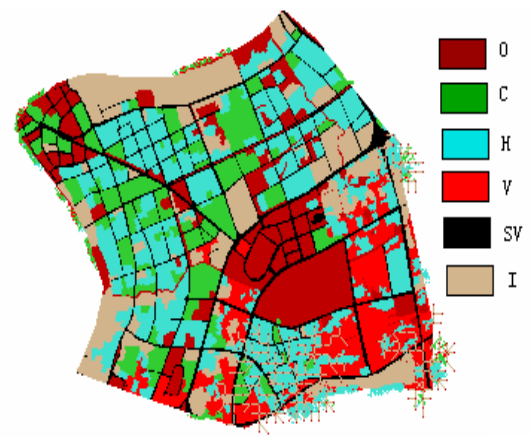

b4) 2020, Lujiazui Model Time: $\mathrm{t}=20$

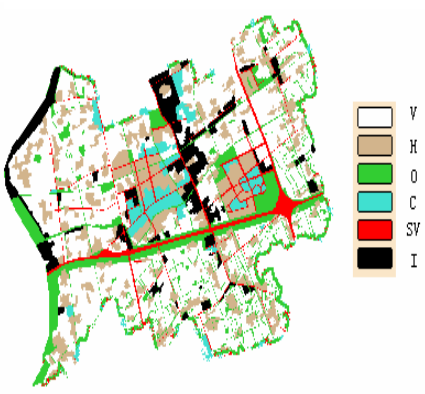

c4) 2020, Sanlin Model Time: $\mathrm{t}=20$ 


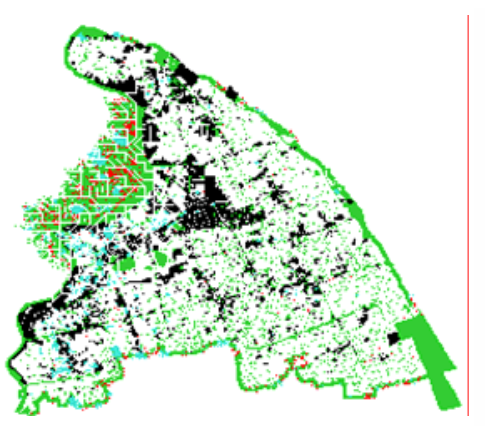

a5) 2050, Pudong

Model Time: $\mathrm{t}=50$

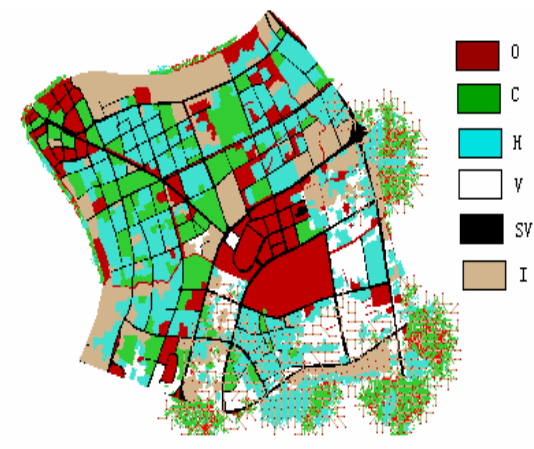

b5) 2050, Lujiazui Model Time: $\mathrm{t}=50$

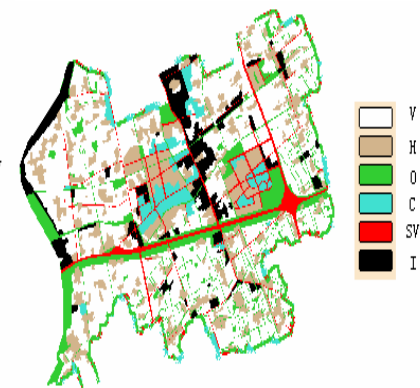

c5) 2050, Sanlin Model Time: $\mathrm{t}=50$

Fig.2: Simulation of urban growth in Pudong New District, Lujiazui Finance \& Trade Zone and Sanlin Town.

\subsubsection{Pudong New District}

(1) The growth mainly occurs at urban periphery in Pudong; (2)The development of housing land mainly occurs at three different areas: northwest area (Donggou Town, Jinqiao Town and Gaoqia Town), southern area of Sanlin Town and central area (Zhangjiang Town and Beicai Town); (3) The development of industry land mainly occurs at southwest of Pudong with lower developing paces at other areas; (4) The development of commerce land mainly occurs at urban periphery and the areas of highway intercross.

\subsubsection{Lujiazui Finance \& Trade Zone}

(1) The growth mainly occurs at urban periphery in Lujiazui; (2) The main types of land use in Lujiazui demonstrate the same development trend as growth points map; the largest growing land type is industry land followed by housing land and commerce land; (3) The development of housing land mainly occurs at three sub-areas: eastern area (periphery of Yangjing Street), southern area (centre of Huamu Street) and southeast area (periphery of Huamu Street); (4) The development of commerce land mainly occurs at northern area (periphery of Meiyuan Street and northwest of Huama Street) while the other areas with lower development paces; (5) The development of commerce land mainly occurs at periphery of Lujiazui which corresponds to the growth of housing land.

\subsubsection{Sanlin Town}

(1) The growth mainly occurs at urban periphery in Sanlin; (2) The three types of land use in Lujiazui demonstrate the same development trend as growth points map; the largest growing land is housing land followed by commerce land and industry land; (3)The three types of land continue its growth on the basis of seeds points while the growth pace is lower than that of Lujiazui.

Apparently, the above characteristics reflect the urban growth trend of the three areas. The predication result of ECA model integrated with GIS accords with law of time decaying which means the longer the predication time, the lower the predication precision. The simulation results of ECA model are not the accurate predication of the urban development but reflect a possibility of urban development of study area. At the present time, there is no a model that enables the precise predication of urban development. Due to the inherent uncertainty and chaos of urban development, there will be no a precise model in the future. The value of CEA model is the ability of assisting geographer and urban planner to understand the urban development characteristics.

The data quality and facticity, and the factors considered by the model will result in decisive influences to a certain degree. In the application of ECA, the introduction of fine data and more strict and rational factors will improve the precision of model predication. However, the facticity and reliability of the ECA model lie on mechanism and process of the model. Therefore, the precision of simulation results can reflect the advantages and disadvantages of the model, but the precision is not the only criterion for the quality of model. Foremost, the key point is whether the model can reflect the inherent laws of urban system or not.

\section{Conclusions}

Using ECA model integrated with GIS, multi-scale urban growth are simulated and predicated for the first time. The simulation and predication results generally reflect the urban development of the three areas at different scales. 
In the process of urbanization, the main driving factors of urban growth vary much from region to region. Analysis of society-economy data and statistical data help us to find the main factors to a certain degree.

The statistical data of the three areas demonstrate that urban growth possesses positive correlativity relationship with population quantity, and possesses approximate positive correlativity relationship with production value. The impact of the relationship is manifested in parameters setting of model: in Pudong New District, the main urban development type is housing land and its developing energy and probability are greater than the other two land use types (commerce land and industry land); in Lujiazui Finance \& Trade Zone, the main urban development type is commerce land and its developing energy and probability are greater than the other two land use types (housing land and industry land); in Sanlin Town, the main urban development type is housing land and its developing energy and probability are greater than the other two land use types (commerce land and industry land). The differences of parameters setting correspond to the modeling principle of ECA model and the development characteristics of the regions.

As a modeling tool, ECA can simulate complex dynamic evolutional phenomena. While the multiscale ECA can simulate and predict the expansion extent and spatial distribution of urban growth. Through the cases study, the practicability of ECA is tested and the application of ECA is developed greatly from mono-scale to multi-scale.

\section{Acknowledgement}

The work described in this paper is substantially supported by Program for New Century Excellent Talents in University (Grant No. NCET-06-0381), Foundation of Shanghai Rising-Star Program (Grant No. 05QMX1456), and funds from the Geographical Spatial Information Engineering Laboratory of SBSM (Grant No. 200618).

\section{References}

[1] R. White and G. Engelen, Cellular automata as the basis of integrated dynamic regional modelling. Environment and Planning B, 24:235-246, 1997.

[2] C.Y. He and N. Okada, Modeling urban expansion scenarios by coupling cellular automata model and system dynamic model in Beijing, China. Applied Geography, 26: 323-345, 2006.
[3] F.S. Chapin and S.F. Weiss, A probabilistic model of residential growth. Transportation Research, 2, 1968.

[4] H. Couclelis, Cellular worlds: a framework for modeling micro-macro dynamics. Environment and Planning A, 17: 585-596, 1985.

[5] H. Couclelis, What rodent populations can teach us about complex spatial dynamics? Environment and Planning A, 20: 99-109, 1988.

[6] H. Couclelis, Macrostructure and microbehavior in a metropolitan area. Environment and Planning B, 16: 141-154, 1989.

[7] H. Couclelis, From cellular automata to urban models: new principles for model development and implementation. Environment and Planning B, 24: 165-174, 1997.

[8] R.W. White and G. Engelen, Cellular dynamics and GIS: modeling spatial complexity. Geographical System, 1:237-253, 1994.

[9] R.W. White and G. Engelen, Cellular automata modeling of fractal urban land use patterns: forecasting change for planning applications Budapest. Eighth European Colloquium on Theoretical and Quantitative Geography, pp.1216, 1993.

[10] Z.C. Cao. Analysis of space-time modeling based on GIS and CA. Annual proceeding of China Association of GIS, China Oversea Association of GIS, Beijing, 1998.

[11] S. Feng, G. Gu and S. Dai, Effects of traffic lights on CA traffic model. Communication in Nonlinear Science and Numerical Simulation, Academic Journal in Electronic Edition. Sponsor: Center of Nonlinear Science, Peking University, 2:120-128, 1997.

[12] F. Wu, Simland: a prototype to simulate land conversion through the integrated GIS and CA with APH-derived transition rules. International Journal of Geographical Information Science, 12: 63-82, 1998.

[13] X. Li and G.A. Yeh, Constrained cellular automata for modelling sustainable urban forms. Scientia Georaphica Sinica, 4: 289-298, 1999.

[14] X.P. Liu, X. Li and L. Chen, An improved minimum-distance method for mining transition Rules of cellular automata. Acta Scientiarum Naturalium Universitatis Sunyateni, 4: 103-106, 2006.

[15] Q.S. Yang and X. Li, Dynamic constrained CA model for simulating complex urban systems. Geography and Geo-information Science, 5:1015, 2006.

[16] C.H. Zhou, Z.L. Sun and Y.C. Xie, Study on geography cellular automat, Science Press, 2000. 
[17] C.F. Wang. Study on urban expansion using remote sensing and cellular automata, Surveying and mapping press, 2001.

[18] M.L. Liu and P. Chen, A prototype of urban simulation model based on the theories and methodologies of cellular automata (CA) and multi-agent system (MAS). Scientia Georaphica Sinica, 3:292-298, 2006. 